\title{
Automatic Pest Detector in Agricultural Farms using Acoustic and Image Wireless Sensor Network Technology
}

\author{
Y. L Malathi Latha, J. Manoranjini
}

\begin{abstract}
India is an agricultural country which depends on agriculture and crops for its economic survival. The production of crops is majorly affected by biotic living organisms, mainly the parasites and pathogens. To handle this continuous monitoring of the crop production is addressed seriously in developed countries. Sensor technology is a promising technology which shows great significance in addressing the agriculture and monitoring based on different parameters. In recent years the advanced wireless sensor networking technology has become popular in pest monitoring and early warning to farmers for timely management of potential pests in agriculture. Image sensors captures pests along with the system which would employ an acoustic device sensor for monitoring the pest's noise level which would notify to the farmer of the specific area where the infestation is occurring.
\end{abstract}

Keywords: Crop Areas, Sensor nodes, Bayesian classifier, Dimensionality Reduction, Feature Extraction, Feature Selection.

\section{INTRODUCTION}

The basic idea to develop an automatic classifier which can classify the pests using their sound was an old technique developed using the available audio recording equipment. However, step by step progress has been made on this problem in the intervening decades. The lack of a robust method in this pursuit can be attributed to ensure to cross a milestone in the field of agriculture. Initially the lack of effective sensors has made data collection difficult. Earlier days acoustic microphones were used. Secondly, ensuring effective quality of data is a challenging task as many researchers have attempted to learn very complicated classification models, especially neural networks. However they were able to use a small set of data for their complex models. To build an efficient robust model we need a large data set.

Existing systems for forecasting the pests and its spreading mostly depends on farmers who personally monitor the crops based on their previous experiences. The drawback of this activity is that not only it is a time consuming and resource wasting activity, but also that one has to wait to detect symptoms, and hence one is unable to detect some harmful pests at an early stage.

Sensor technology is considered to be highly effevtive to farmers to monitor crops efficiently, potentially and remotely detect destructive insects / pests and to prevent their relevant disease in early stage. Earlier technology used was sound recorder which was able to identify calculated number of pests. We propose a novel technology which combines the use of image and acoustic sensors. The Image and Sound sensors collect the different kinds of pests images and sounds and transmits it to our pest detector which would employ a Bayesian classifier which can classify the harmful and non harmful pests found in that particular area and inform the farmer with an alarm alert message when there are harmful pests.

A principled method is designed to incorporate additional information into the classification model. This additional information can be an easy-to-obtain as the time-of-day, rhythmic sounds, count of the flapping of wings etc.

From the data collected we work our novel methodology which results in a simple, accurate and robust classifier. In summary, flying pest detection can be moved further from the research lab and deployed in the real world. In addition to this the sensors and software presented in this work will provide researchers worldwide robust tools to accelerate their research.

\section{PROPOSED WORK}

We can classify our work as follows,

To develop a methodology for noninvasive sensors to accurately and automatically identify flying insects and pest identification by incorporating.

Detection algorithm which uses a $0.0 .5 \mathrm{sec}$ long sliding window to slide through the recording.

i) Bayesian Classifier.

A. To develop an efficient classifier which has i) a training phase, where it learns the nature of the pests and ii) a classification phase where, given the new feature of the "unknown sound and image", it computes the probability of observing the feature in class using the density functions learned in the previous step.

B. To evaluate the efficiency of new methods developed using the image and sound sensors based on computation speed and period of time. 


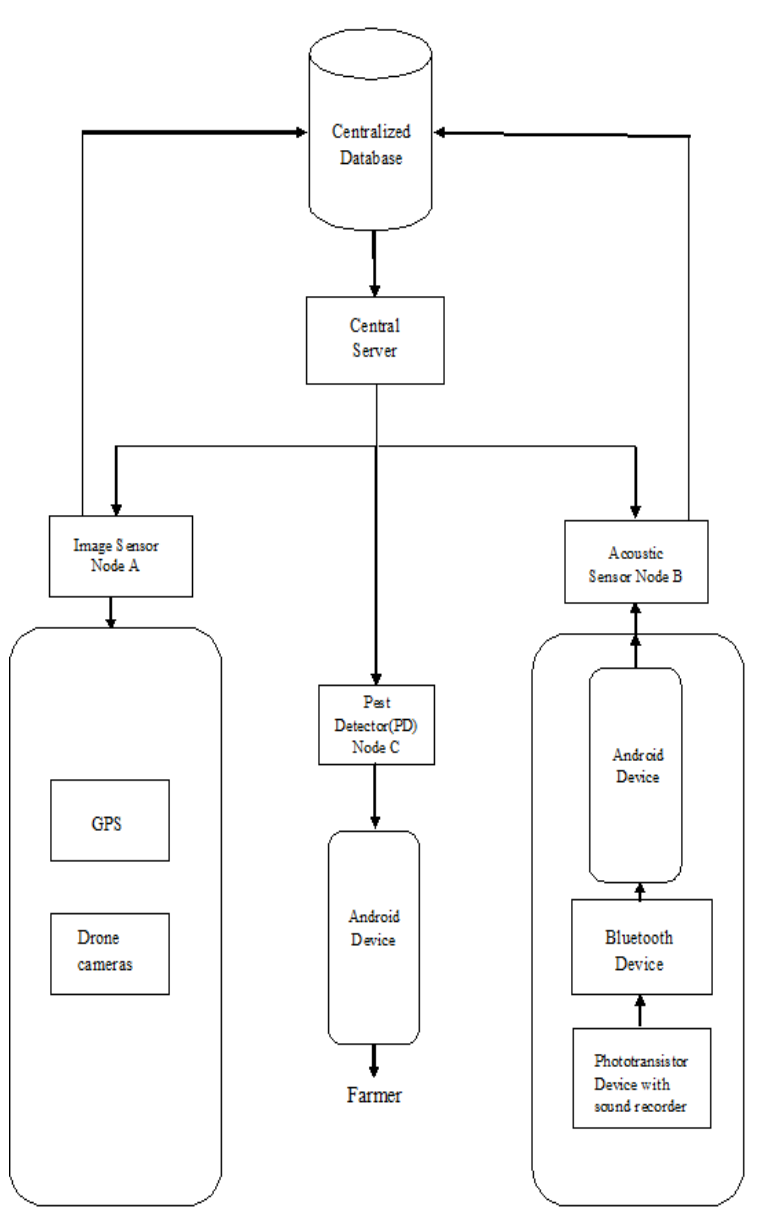

Fig. 1. Architecture of Pest Detector

\section{METHODOLOGY}

Our methodology could be elaborated. We have several phases like, Pre processing, Dimensionality Reduction, Feature Extraction, Feature Selection, Detection Algorithm and finally a classification phase.

\section{A. Pre-processing}

Hyperspectral image which is particularly suitable for vegetation identification, monitoring and assessment, is given as input. Naturally, all images and sound recorders contain noise that occurs due to errors caused in the sensors, irregular illumination and atmospheric conditions. Hence, the preprocessing is required to remove these noises.

\section{B. Dimensionality Reduction}

Hyperspectral data contain a massive amount of information; this redundant data limits the processing speed. To remove these redundant data, dimensionality reduction technique is used. Some of the dimensionality reduction techniques are Principal Component Analysis (PCA), Linear Discriminate Analysis (LDA) and Independent Component Analysis (ICA).

\section{Feature Extraction}

The pest classification using hyperspectral data mainly consists of three types of feature. The spectral features, spatial features and spectral indices which are obtained by calculation between different spectral bands that are sensitive to the biological parameters of the vegetation are also extracted.

\section{Feature Selection}

The combination of the spectral features, spatial features, as well as spectral indices forms a massive amount of information or features. Among the extracted features, the significant features are selected using feature selection techniques. Some of the feature selection techniques are information gain, gain ratio etc.

\section{E. Detection Algorithm}

1. Use a $0.05 \mathrm{sec}$ long sliding window to slide through the recording

2. Compute the fundamental frequency of the current window.

3. If the fundamental frequency is within the range of 100 $\mathrm{Hz}$ to $1,200 \mathrm{~Hz}$ do step 4.

4. Extract the 1-sec long audio clip centering at the current window from the recording; apply a digital filter to remove the noise in the clip and save the filtered audio.

5. Move the sliding window to the point that immediately follows the extracted audio clip.

6. If the fundamental frequency is NOT within the range of $100 \mathrm{~Hz}$ to $1,200 \mathrm{~Hz}$, simply move the sliding window $0.01 \mathrm{sec}$ forward.

7. Repeat step 4 to 6 until the sliding window reaches the end of the recording.

\section{F. Classification}

The classification method based on the construction and optimization of features can enhance the identification of the variety of pest classes. The classification algorithms would be used for pest detection are Bayesian classifier which includes.

\section{G. Training Phase}

Use the $\mathrm{k}$ nearest neighbours algorithm with density estimation approach to learn the posterior probability distribution using the sound feature. We classify and train few datasets.A random sample of insect are collected and their effective sound variance ranging in all hertz are for few varieties. Every insect sound, is computed based on its frequency spectrum using the Discrete Fourier Transform (DFT). Consider only the data points corresponding to the frequency range: $100 \mathrm{~Hz}$ to $2,000 \mathrm{~Hz}$. The omitted ones are then used in the classification as the "representative" of the insect sound.

\section{H. Classification Phase}

Calculate the Euclidean distance between the omitted spectrum of the unknown object and all the omitted spectrums in the training dataset. Find the top $\mathrm{k}$ nearest neighbors of the unknown object in the training dataset. Compute the posterior probability of the unknown insect sound and image belonging to a class as the fraction of the top $\mathrm{k}$ nearest neighbors which are labeled as class. A Classification is done based on the unknown object to the class that has the highest posterior probability. Finally, post processing is done on the classified output to extract the crop areas.

Performance analysis of various computational methods and techniques to formulate a computational system are evaluated. 
Classification and development of a software system require the knowledge of programming languages and visually appealing front end design. These algorithms can be implemented using the ENVI, MATLAB and WEKA tools. Statistical analysis of the proposed algorithms and techniques will be performed using SPSS and R software.

\section{SENSOR TECHNOLOGY}

We have introduced two sensor technology in our proposal one is a high image sensor and another is a sound detection sensor and we also use a third sensor which is our pest detector. Let us brief into more about them.

\section{A. High-image Sensors}

The vegetational plants and soil reflect a certain amount of light energy to the atmosphere which can also be detected using a phototransistor. This light energy is generally referred to as the spectral signature. When we use high-image sensors it can easily capture the spectral signature of every plant or crop and record it. These images can capture any gamma rays, ultraviolet rays, $\mathrm{x}$-rays and infrared. The image data produced is multispectral (more than 3 bands) or hyperspectral (hundreds of bands). These high resolution images give the physical and chemical details of the plant from hundreds of kilometers away. Our sensor, analyzes the images, and accurately detects multiple types of pests. We also use drone technology to obtain the features of the pests in detail.

\section{B. Sound Detection Sensors}

The use of sound sensors are an effective way to identify more pests which are generally very small in size and cannot be captured by the current image sensor or failed to be captured by the image sensor. These wireless sensors have antennas which capture the sound waves. We place our sensors in strategic points across the agricultural land. These sensors capture sound generated by pests. They record the noise levels of that place for a certain period and after a short period; the collected data is fed into a computer so as to analyze it. Places with harmful pests usually have higher sound waves than the rest.

We use an effective optical sensor which can be used to record the "sound" of pests flying from meters away, with complete invariance to wind, noise and ambient sounds. These sensors are able to effectively and efficiently record millions of training instances, than all previous efforts combined.

\section{Pest Detector}

Our third sensor is our pest detector. The data collected from the image sensor and sound sensors over a period of time is fed into the detector sensor which is generally a computer or an android device. This sensor maintains a threshold for images and sounds of various pests. When there is a increase above the threshold value the image and sound is compared with the datas from the database to identify the exact pest that is currently affecting the farmland. Based on this the detector sents an alert message to the farmer so that he can be aware of the pests and identify it and eradicate it and avoid disaster to his crops.

\section{PERFORMANCE ANALYSIS}

Table- I: Performance Table

\begin{tabular}{|l|l|}
\hline \multicolumn{1}{|c|}{ Species } & \multicolumn{1}{c|}{ Classification Accuracy } \\
\hline Stem borer & $83.01 \%$ \\
\hline Boll worm & $85.15 \%$ \\
\hline Thrips & $93 \%$ \\
\hline Rootborer & $90.11 \%$ \\
\hline Weevil & $88.25 \%$ \\
\hline Tarsalis & $90.01 \%$ \\
\hline Drosophila & $93 \%$ \\
\hline Musca Domestica & $95 \%$ \\
\hline
\end{tabular}

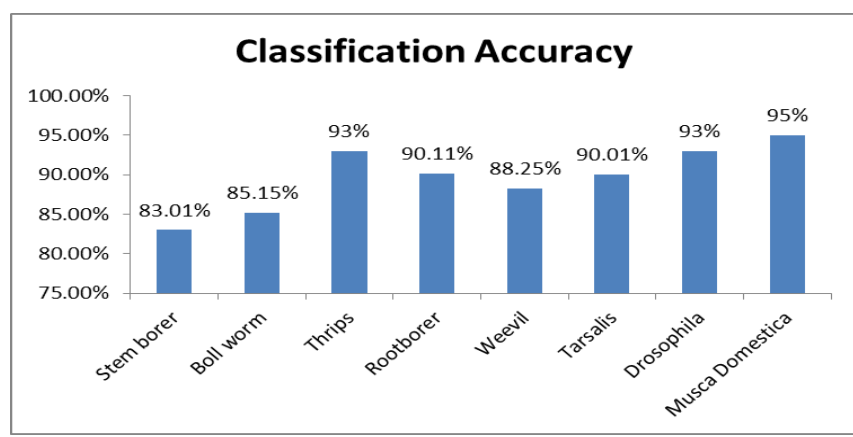

Fig. 2.Classification Accuracy

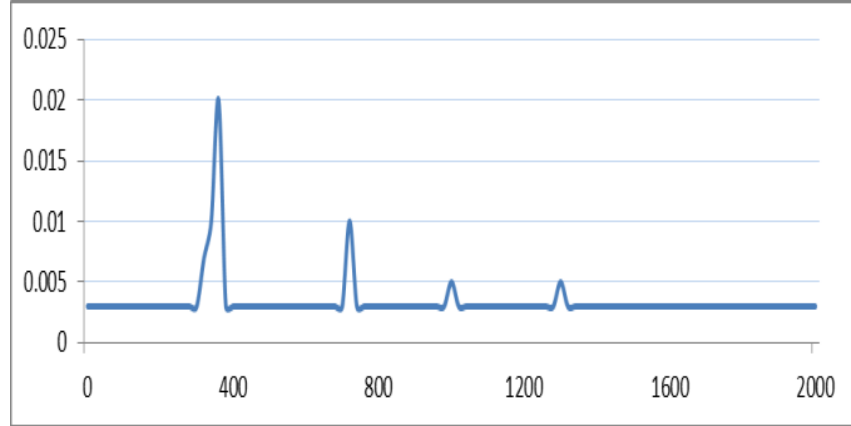

Fig. 3.Frequency spectrum of pests

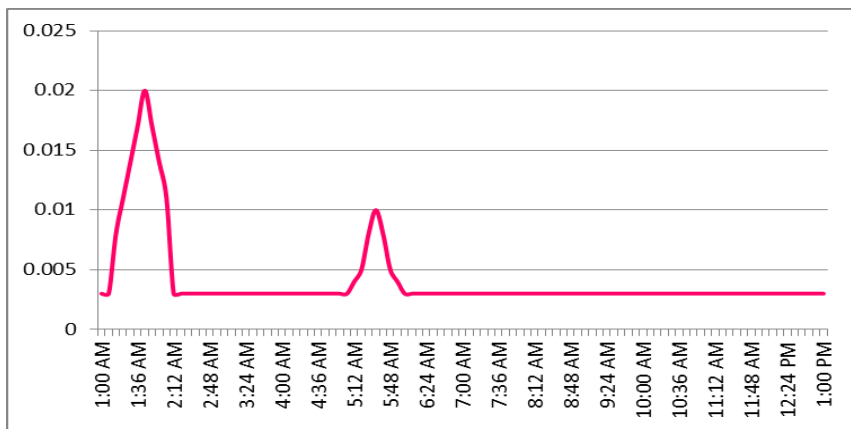

Fig. 4.Observations over a period of 1 month

\section{ACKNOWLEDGMENT}

This research was supported by Swami Vivekananda Institute of Technology. We are immensely grateful to the management of our college for their continuous encouragement and support.

We would also like to show our gratitude to our Principal for sharing his pearls of wisdom with us during the course of this research. We thank our dean, for his assistance with the technique and methodology that greatly improved the manuscript. 


\section{REFERENCES}

1. Patil P., Vidya H, Patil S, Kulkarni U. Wireless Sensor Network for Precision Agriculture, International Conference on Computational Intelligence and communication systems, 2011.

2. Mampentzidou I, Karapistoli E AA. Economides: Basic Guidelines for deploying Wireless Sensor Networks in Agriculture: The 4th International Workshop on Mobile Computing and Networking Technologies, 2012.

3. Cheng J, Liang G. Double barrier Coverage in Dense sensor Networks, Journal of computing and Technology, Jan, 2008.

4. Tripathy AK, Adinarayana J, Sudharsan D, Vijayalakshmi K, Merchant S, Desai UB. data processing and Wireless Sensor Network for Groundnut Pest / Disease Interaction and Prediction - A Preliminary Study ISSN 2013; 5:2150- 7988.

5. Panchard J. Wireless Sensor Networks for Marginal Farming in India. PhD Thesis. Ecole Polytechnique Lausanne: EPFL, 2008.

6. Vinayak SV, Dr Mrs Apte SD. Real Time Monitoring of Agri-Parameters Using WSN for Precision Agriculture, International Journal of Advanced Research in computing and Software Engineering, 2013, 3(9). 99 Journal of Entomology and Zoology Studies.

7. Wang N, Zhang N, Wang M. Wireless Sensors in agriculture and food industry- Recent Development and future perspective, Elsevier Computer and electronics in Agriculture,2006.

8. Zhand N, Wang M, Wang N. Precision Agriculture- A worldwide Overview, Elsevier Computer and Electronics in agriculture,2002.

9. Lee WS, Alchanatis V, Yand C, Hirafuji M, Moshou D, Li C. Sensing Technologies for precision specialty crop production; Elsevier Computer and Electronics in agriculture, 2010

10. Patil P, Vidya H, Shreedevi P, Umakant K. Wireless Sensor Network for Precision Agriculture, International Conference on Computational Intelligence and and communication system,IEEE,2011.

11. Srivastav N, Chopra G, Jain P, Khatter B. Pest Monitor and system using WSN with special referance to Acoustic Device;ICEEE27thJan,2013.

12. Srinivas S, Harsha KS, Sujatha A, Kumar N. Efficient protection of palms from RPW Larvae using WSN: IJCSI 2013,10(3):2.

13. Mohammad A Al-Manie, Mohammad I Alkanhal. Acoustic Detection of the Red Phoenix dactylifera Weevil; IJERECE 2007

14. Mauro prevostini. Wireless Sensor Network for Pest Control, Commission for Technology and innovation CTI, 2011.

15. Charlet LD, Olson D, Glogoza PA. Biological Control of Insect and Weed Pests in North Dakota Agriculture. NDSU extension ,2002.

16. Antinelli A, Coletta A, Pucci C. Economic comparison of Traditional, Guided and Biological Pest Control in Italian Potato Production., Workshop on pesticides, policy measure to regulate environmental impacts from agriculture. Wageningen, The Netherland, August 24-27, 1995.

17. Akhtar W, Sengupta D, Chowdhury A. Impact of Pesticides use in agriculture: Their benefits and hazards, Interdisc Toxicol,2009.

18. Gianessi L, Reigner N. Barriers to widespread conversion from Chemical Pest Control to non-Chemical Methods in US Agriculture, Pest and Nutrient Management. Track 1.

19. Holt KM. Opit G, Nechols JR, Margolies DC, Williams KA. Comparing chemical and biological control strategies for Twospotted Spider Mites in mixed production of ivy-leaved geranium and Impatiens, Hort Technology, Research Report,2007.

20. William GM, Linker HM, Waldvogel MG, Leidy RB, Schal C. Comparison of Conventional and Integrated Pest Management Programs publicly Schools, Entomological SocietyofAmercia,2005.

21. Beech CJ, Koukidou M, Morrison NI, Alphey L. Genetically Modified Insects: Science, Use, Status and Redulation, Collection of Biosafety Reviews International Center of gene-splicing and Biotechnology (ICGEB), 2012,

22. Thresher RE. "Genetic Options for the Control of Invasice Vertebrate Pests: Prospects and Constraints" Managing Vertebrate Invasive Species, 2007.

23. Vennila S, Ramasundram P, Raj S, Kairon MS. Cotton IPM and Its Current Status", CICR Technical Bulletin \#, 2000.

24. Singh OV, Ghai S, Paul D, Jain RK. Genetically Modified Crops: success, safety assessment and public concern, Appl. MicrobiolBiotechnol,2006.

25. Ahmad B, Anjum R, Ahmad A, Yousaf MM, Hussain M, Muhammad W. Comparison of various methods to regulate pomace fly (Carpomyia Vesuviana) on BER. Pak. Entomol2005.
26. Aziz MA, Hasan M, Ali A, Iqbal J. Comparative efficacy of various strategies for management of spotted Bollworms, Earias spp on Okra, Pakistan Journal of Zool, 2012

27. Mehdipour F, Nunna KC, Murakami KJ. a sensible cyberphysical system-based solution for pest control. International conference on Green computing and communication and IEEE internet of thinds ad IEEE Cyber Physical and Social Computing, 2013.

28. Mahmood R, Rehman A, Ahmed M. Prospects of biological control of citrus insect pests in Pakistan, Journal of Agric. Research,2014.

\section{AUTHOR PROFILE}

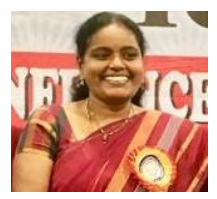

Y. L Malathi Latha received the Doctoral degree from Jawaharlal Nehru Technological University Hyderabad, India. Currently she is a Head \& Associate Professor in Swami Vivekananda Institute of Technology (SVIT), Hyderabad, India. She has published over 10 papers in International and National Journal/Conferences. Hers research interest includes Biometrics, Information fusion, Image Processing, and pattern recognitions.

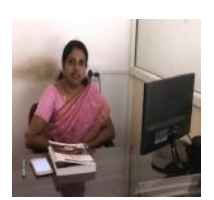

J. Manoranjini received the Doctoral degree from Anna University, TamilNadu, India.Currently she is the Associate professor in Swami Vivekananda Institute of Technology (SVIT), Hyderabad, India. She has published over 10 papers in International and National Journals/Conferences.Her research interest includes Adhoc Networks, MANETS, Wireless Sensor Networks, Network Security. 